\title{
Rayleigh scatter in kilovoltage x-ray imaging: is the Independent Atom Approximation good enough?
}

\author{
G Poludniowski, P M Evans and S Webb \\ Joint Department of Physics, \\ Institute of Cancer Research and Royal Marsden NHS Foundation Trust, \\ Downs Road, Sutton, Surrey, SM2 5PT UK \\ E-mail: Gavin.Poludniowski@icr.ac.uk
}

\begin{abstract}
Monte Carlo simulation is the Gold Standard method for modelling scattering processes in medical x-ray imaging. General-purpose Monte Carlo codes, however, typically use the Independent Atom Approximation (IAA). This is known to be inaccurate for Rayleigh scattering, for many materials, in the forward direction. This work addresses whether the IAA is sufficient for the typical modelling tasks in medical kilovoltage x-ray imaging. As a means of comparison we incorporate a more realistic 'interference function' model into a custom-written Monte Carlo code. First, we conduct simulations of scatter from isolated voxels of soft-tissue, adipose, cortical bone and spongiosa. Then, we simulate scatter profiles from a cylinder of water and from phantoms of a patient's head, thorax and pelvis, constructed from diagnostic-quality CT data sets. Lastly, we reconstruct CT-numbers from simulated sets of projection images and investigate the quantitative effects of the approximation. We show that the IAA can produce errors of several percent of the total scatter, across a projection image, for typical x-ray beams and patients. The errors in reconstructed CT-number, however, for the phantoms simulated, were small (typically $<10 \mathrm{HU}$ ). The IAA can therefore be considered sufficient for the modelling of scatter-correction in CT imaging. Where accurate quantitative estimates of scatter in individual projection images are required, however, the appropriate interference functions should be included.
\end{abstract}

\section{Introduction}

In this paper we limit the term 'kilovoltage x-ray imaging' to refer to the imaging of an extended object with a broad-area kilovoltage x-ray beam, with the aim of measuring the primary unattenuated signal deposited in a detector. Scatter is to be considered unwanted and in need of quantification. The capability to accurately model scatter in medical x-ray imaging is desirable as it allows the optimization of sources, geometries and detectors such that the signal due to scatter is minimized. It also, potentially, allows the calculation of scatter signal in, and its subtraction from, a measured image. The Gold Standard method for scatter simulation in x-ray imaging is the Monte Carlo (MC) method (see e.g. Chan and Doi 1983). Currently there are several general-purpose MC codes readily available for conducting calculations e.g. EGSnrc (Kawrakow and Rogers 
2006), GEANT4 (Agostinelli et al 2003), PENELOPE (Salvat et al 2003), MCNPX (Waters et al 2007). The precise way in which each interaction is modelled, and the however, the photo-electric effect, Compton scattering and Rayleigh scattering must all be considered. Rayleigh (or coherent) scatter is an important contribution to the total scatter signal in kilovoltage x-ray imaging. Recently, it has been highlighted that the Rayleigh process is the dominant contribution to spatial-modulations in the scatter of general-purpose MC codes, and many more specialized codes, that they operate in the Independent Atom Approximation (IAA). For the Rayleigh interaction, this approximation fails at forward scattering angles. This is because of the disregard of extra-atomic interference between electrons in the bulk material. This weakness is recognized by those releasing state-of-the-art MC codes: e.g. in the EGSnrc package it is possible to specify your own Rayleigh form factors. In fact, quite precise information regarding the angular distributions of Rayleigh scattering from biological materials is known, due to experimental studies (Narten and Levy 1971, Kosanetzky et al 1987, Royle and Speller 1991, Peplow and Verghese 1998, Elshemey et al 1999). This (1983) discussed the effect in detail, for scattering from water, and demonstrated the observability of the resulting diffraction peaks for a polyenergetic beam. Persliden and Carlsson (1997), for water, explored the consequences of such interference for diagnostic x-ray imaging using MC methods. Other authors have given specific focus to the importance of coherent scattering in mammography e.g. Peplow and Verghese (2000) or Taibi et al (2000). More recently, Cardoso et al (2003) have made general observations based on MC simulations, again, for water only. These last authors argued that interatomic interference is important for relatively thin samples and therefore primarily in mammography. We feel, however, that the case for the defence of the IAA is incomplete. Firstly, we are aware of no simulation studies investigating the limitations of the IAA for scatter from large heterogeneous objects, or, indeed, objects of anthropomorphic form and composition such as a head, thorax or pelvis. Secondly, we are aware of no studies examining the consequences for CT imaging of such objects. In this paper we address these issues. A custom-written MC program, validated elsewhere (Poludniowski 2009a), was modified for this purpose. This program has the advantage that it is optimized for simulating imaging and hence fast; it is also trivial to modify the Rayleigh scattering data. Tartari et al (2002) have provided experimental Interference Functions (IFs) for several biological materials: water, fat, bone matrix and bone mineral. Those authors have suggested that these four materials can be used as a basis set for constructing a realistic model of a human patient. The Tartari data therefore provides an opportunity for modelling anthropomorphic bodies using a $\mathrm{MC}$ code and the Interference Function Model (IFM). The range of imaging and detector geometries and $\mathrm{x}$-ray beam qualities used throughout the field of medical x-ray imaging is enormous. It could not be comprehensively covered here. Instead we focus on one particular setup 
80

reflecting an x-ray unit in our radiotherapy department: a Synergy XVI Cone-Beam CT (CBCT) unit (Elekta Limited, Crawley, UK). This choice is a good candidate for several reasons. Firstly, previous modelling work has been carried out on this unit in our group (Poludniowski et al 2009a, Roberts et al 2008). Secondly, it is known that there is a large amount of scatter generated in $\mathrm{CBCT}$ and that this scatter leads to a quantitative change in reconstructed CT-number (Siewerdsen and Jaffray 2001). Finally, although an air gap between patient and detector such as is employed in this unit leads to a degree of scatter rejection, it also leads to an increase in the proportion of scatter due to the Rayleigh interaction (Johns and Yaffe 1983). The theory of x-ray interference effects is well-known in the field of x-ray diffractography and crystallography, however, as this paper is aimed at a different audience, some theoretical background is included. From the starting point of the observations of the failure of the IAA in the diffraction imaging of small samples, the three core questions this paper answers are:

- For the typical source-detector geometry and polyenergetic x-ray source modelled, does the use of the IAA introduce appreciable errors into the predictions of scatter signal in the detector?

- What are the magnitudes of discrepancies for typical extended and anthropomorphic objects?

- Do any such discrepancies lead to non-negligible differences in CBCT reconstructions?

We note that the various types of scatter imaging and tomography (see e.g. Harding and Schreiber 1999) are not under direct consideration in this work.

\section{Theory and methods}

\subsection{Rayleigh scattering}

In a discussion of the scattering of x-rays, a quantity of fundamental significance is:

$$
x=\frac{E}{h c} \sin \frac{\theta}{2},
$$

where $E$ is the photon energy, $\theta$ is the scattering-angle, $h$ is the Planck constant and $c$ is the speed-of-light. The momentum exchange, $q$, in the scatter, is: $q=2 h x$. In general, a material can be considered to consist of a large number of 'units', or groups of atoms, arranged in a particular manner in relation to each other. In such a case, a fundamental result is that the Rayleigh scattering differential cross-section (RS) can be approximated by the Thomson differential cross-section for scattering from a free electron (Th) multiplied by a unit scattering power. Thus,

$$
\frac{d \sigma_{R S}}{d \Omega}=\frac{d \sigma_{T h}}{d \Omega} I(x),
$$

where $I(x)$ is the (spherically symmetric) unit scattering power. We consider only the case where, due to symmetry in the geometrical structure or averaging over unit 
orientations, such as occurs for amorphous materials, we can reduce the unit scattering power to a function of one scalar variable, $x$. This unit scattering power, in turn, can be decomposed into the product of the square of a Form Factor (FF) for the scattering unit, $f^{2}(x)$, multiplied by an Interference Function $(\mathrm{IF}), \mathcal{F}(x)$ (Guinier 1994):

$$
I(x)=f^{2}(x) \mathcal{F}(x) .
$$

The FF encodes the interference effects between electrons inside a particular unit. The IF includes the effect of interference between electrons within neighbouring units. For an isotropic substance, it can be written as (Guinier 1994):

$$
\mathcal{F}(x)=1+\frac{1}{v} \int_{0}^{\infty}(g(r)-1) \frac{\sin 4 \pi r x}{4 \pi r x} 4 \pi r^{2} d r,
$$

where $g(r)$ is the distribution function that describes the statistical configuration of scattering units in relation to each other and $v$ is the average volume per unit.

\subsection{The interference function}

The data that would be required to tabulate the molecular form factors and interference functions of all necessary materials, under all thermodynamic conditions, are enormous. Additionally, much of this information is unknown. So, for practical reasons, in generalpurpose MC codes, any material is usually considered to be a collection of independent atoms, in the appropriate ratios. Thus the distortion of the electron wave-functions and therefore FFs by bonding, and, the interference between any two scattering units is ignored. Let us illustrate when this approximation fails by considering the case of water. The IAA unit scattering power for water, $I_{\text {water }}^{I A A}(x)$, is:

$$
I_{\text {water }}^{I A A}(x)=2 f_{H}^{2}(x)+f_{O}^{2}(x),
$$

where $f_{H}(x)$ and $f_{O}(x)$ are the atomic form-factors of hydrogen and oxygen, respectively. This function is plotted in figure 1 (a) (solid black line). It is also possible to calculate an approximate molecular FF taking into account bonding and interference between electrons in the constituent three atoms (Morin 1982). The unit scattering power for such an Independent Molecule Approximation (IMA) calculation, $I_{\text {water }}^{\text {IMA }}(x)$, is plotted in figure 1 (dashed black line). An experimentally determined scattering power, $I_{\text {water }}^{E x p}$, is also shown (solid grey line: Tartari et al 2002). Both the IAA and IMA approximations disagree with experiment at small $x$. In fact, the IMA provides a poorer agreement than the IAA. The water IF must therefore have a dramatic effect on the low$x$ behaviour of the scattering. The Rayleigh scattering of x-rays from water is dominated by the presence of the oxygen atoms. We suggest therefore that the scattering power can be approximated by the IMA FF multiplied by the IF calculated from the distribution of oxygen separation, $g_{O O}(r)$. This latter function has been estimated from numerical calculations (Soper 2000) and is plotted in figure 1 (b). The series of local maxima in the function arise because water possesses a loose tetrahedral structure even as a liquid (Head-Gordon and Hura 2002). An improved approximation for the scattering power 
of water, $I_{\text {water }}^{C a l}$, is then:

$$
I_{\text {water }}^{\text {Cal }}(x)=I_{\text {water }}^{\text {IMA }}(x) \mathcal{F}_{O O}(x) .
$$

This scattering power is also plotted in figure 1 (a) (dotted black line) and provides good agreement with the data. This result demonstrates that in some circumstances, even an amorphous material must be treated as a material rather than individual atoms or molecules. It is impractical to conduct a similar analysis for every material of practical significance, since many materials require a more refined treatment and much of the information on material structure is unavailable. It is, however, practical to move beyond the IAA for a specific task, such as medical x-ray imaging, in which a reduced set of data is needed and the relevant unit scattering powers can be measured.
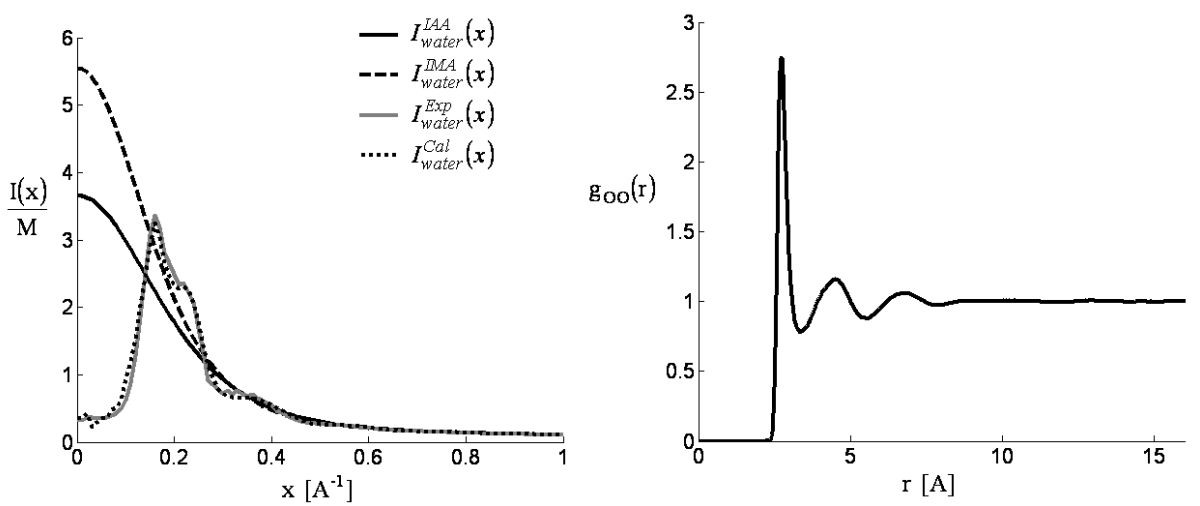

Figure 1. (a) IAA, IMA, experimental (Exp) and calculated (Cal) scattering powers, normalized by molecular mass $(\mathrm{M})$, as functions of $x$; (b) the function $g_{O O}(r)$ as a function of $r$ (1 atmosphere and $298 \mathrm{~K})$.

\subsection{Monte Carlo modelling and phantom data}

A MC code developed in-house was adapted for this study. This code, written in Fortran 95, is described in detail elsewhere (Poludniowski et al 2009a). In this previous study the program was validated and applied to the problem of scatter-correction in Cone Beam CT (using the IAA model). In brief: the Compton angular distribution was taken to be that of the Klein-Nishina formula modified by the incoherent scattering function tabulated by Hubbell et al (1975); the Rayleigh angular distribution was taken to be that of the Thomson formula modified by the form-factor (Hubbell et al 1975) and appropriate IF (Tartari et al 2002). Attenuation coefficients were taken from the NIST $\mathrm{XCOM}$ database (Berger et al 2005). Forced detection was used to increase detection efficiency at the simulated flat-panel detector. The approach has features in common with the 'collision density estimator' approach of Persliden and Carlsson (1997). Quasirandom numbers were used in combination with pseudo-random number to further increase calculation efficiency. The primary ray-projections through a phantom were calculated by ray-tracing. The use of this fast and reasonably accurate model allows 
us to explore consistently the effect of one particular feature: the effects of the IF on observed scatter.

In this study five materials were simulated: air, soft-tissue, adipose, cortical bone and spongiosa. Table 1 provides the assumed composition of these materials in terms of the basis materials of the Tartari data. The proportions shown for adipose, cortical bone and spongiosa were derived from fitting the atomic proportions of $\mathrm{C}, \mathrm{N}, \mathrm{H}$ and $\mathrm{O}$ quoted in ICRU Report 44 (ICRU 1989). The phantoms simulated were either isolated voxels of tissue, voxelized water cylinders or voxelized phantoms representing patient anatomy. Three anthropomorphic phantoms were constructed from CT planning scans acquired in our radiotherapy department: head (512x512x100 voxels), thorax (512x512x198 voxels) and pelvis (512x512x73 voxels). The digital units of the DICOM files were equated to densities and the voxels partioned into one of five materials based on density. The precise proportion of materials and voxel densities are not of prime importance. Of more importance is the fact that the anthropomorphic phantoms are representative of typical spatial variations in patients and typical compositions. For projection images through these anthropomorphic phantoms, scatter was calculated to a set of $128 \times 128$ points in the detector and linearly interpolated to provide $256 \times 256$ scatter images.

The imaging system modelled was a Synergy XVI CBCT system (Elekta Limited, Crawley, UK). Figure 2 illustrates the acquisition geometry. The source-to-axis distance (SAD) was set to $100 \mathrm{~cm}$ and the source-to-detector distance (SDD) to $153.6 \mathrm{~cm}$. The flat-panel was modelled as a $\mathrm{CsI}(\mathrm{Tl})$ detector using the energy response curves of Roberts et al (2008). The detector width, $W$, was $41 \mathrm{~cm}$. The incident x-ray beam was calculated using the SpekCalc software utility (Poludniowski 2007, Poludniowski et al 2009b) and half-value-layer data from an XVI unit in our department $(7.0 \mathrm{~mm} \mathrm{Al}$ at $120 \mathrm{kV})$. Other tube potentials were simulated with the inferred filtration $(7.8 \mathrm{~mm} \mathrm{Al})$ held constant. CT reconstructions were performed using an implementation of the Feldkamp algorithm (Feldkamp et al 1984). All simulations except those for the CT reconstructions were performed on a desktop PC with a single $3.4 \mathrm{GHz}$ Pentium $4 \mathrm{CPU}$ and $2 \mathrm{~GB}$ of RAM. Because of the time-intensive nature of simulating multiple projections, the projections for CT reconstructions were performed on a 50 CPU cluster of X86-64 processors. All code was written in Fortran 95 and compiled with the gfortran compiler (GNU GCC). Figure 3 illustrates the close agreement between the in-house $\mathrm{MC}$ program and the scatter predictions of the state-of-the-art BEAMnrc code (Rogers et al 2004), within the IAA model, for a test case in the Synergy XVI geometry. A $100 \mathrm{kV}$ x-ray source spectrum was used in each case and the test phantom was a $10 \times 10 \times 10 \mathrm{~cm}^{3}$ cube of water placed at the origin $(O)$. The energy fluence at the detector is plotted along a central axis of the detector.

\section{Results and discussion}

The scatter from isolated voxels of material placed at the origin of the XVI imaging geometry, were simulated for a narrow incident pencil beam. Figure 4 (a) to (h) show 
Table 1. Tissue composition in terms of the basis set of Tartari et al (2002).

\begin{tabular}{|c|cccc|}
\hline Material & Water [\%] & Fat [\%] & Bone matrix [\%] & Bone mineral [\%] \\
\hline Air & 0.0 & 0.0 & 0.0 & 0.0 \\
Soft-tissue & 100.0 & 0.0 & 0.0 & 0.0 \\
Adipose tissue & 27.9 & 72.1 & 0.0 & 0.0 \\
Cortical bone & 25.9 & 0.0 & 16.4 & 57.7 \\
Spongiosa & 33.0 & 38.4 & 8.2 & 20.4 \\
\hline
\end{tabular}

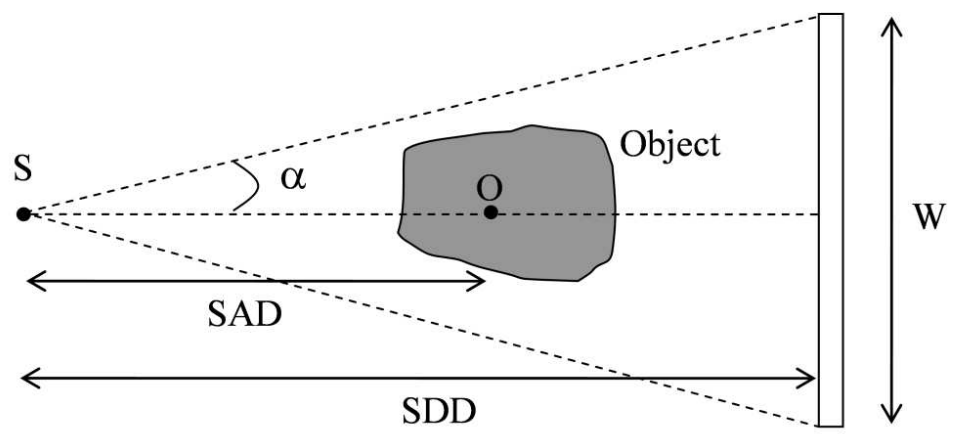

Figure 2. Illustration of the imaging geometry.

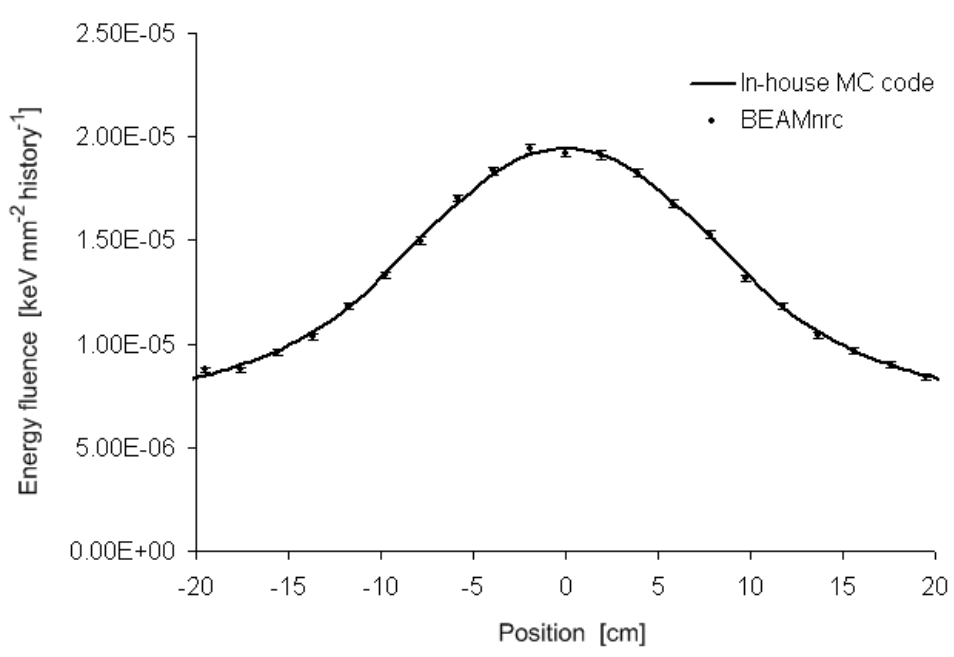

Figure 3. A comparison of the scatter fluence profiles predicted by the in-house code and BEAMnrc along a central-axis of the detector. The BEAMnrc data points were calculated with $4 \times 10^{7}$ histories with bound Compton scattering and Rayleigh scattering enabled; the XCOM photon data were used (Berger et al 2005). 
scatter images produced by $0.5 \times 0.5 \times 0.5 \mathrm{~cm}^{3}$ voxels of soft-tissue, adipose, cortical bone and spongiosa. A $100 \mathrm{kV}$ x-ray beam was simulated with $10^{4}$ photon histories per image (taking a few seconds computation). We observe that the spatial variation in scatter modulates over a region several $\mathrm{cm}^{2}$ in size. Within this region, even for this polyenergetic beam of relatively high energy, the differences between the predictions of the IAA (left column) and IFM (right column) models, are obvious. Associated with each image is a profile of the scatter signal along the central horizontal line (solid lines). The 1st order Rayleigh (dashed lines) and Compton scatter (dotted line) contributions are also shown (the latter of which is identical for the IAA and IFM models). Note the higher-spatial frequencies present in Rayleigh scatter contributions for the more realistic IFM simulations, compared to the conventional IAA predictions. Therefore the effects observed from the diffraction imaging of biological materials are still apparent for typical medical x-ray imaging geometries and beams.
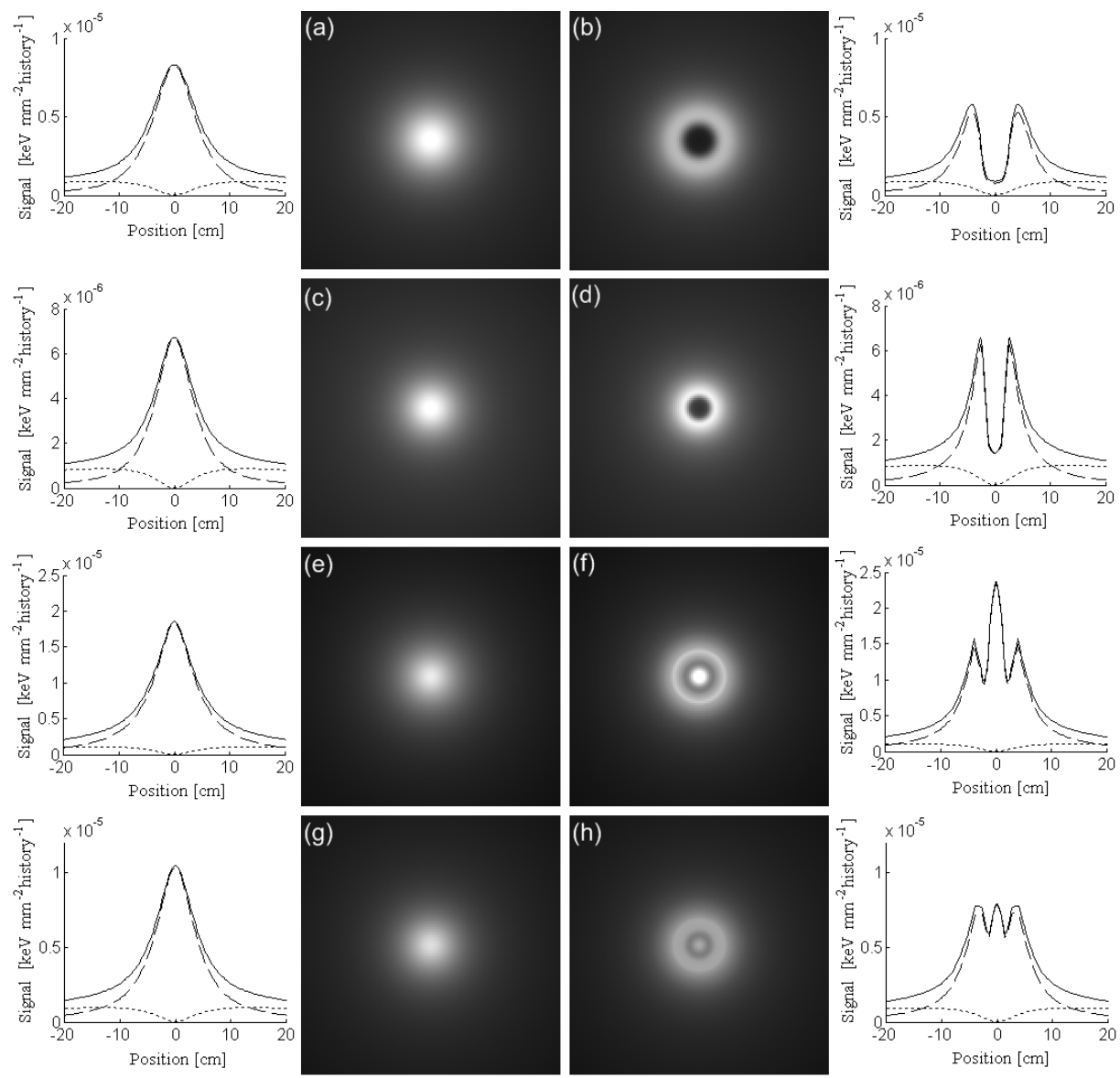

Figure 4. $41 \times 41 \mathrm{~cm}^{2}$ scatter images due to the isolated voxels (left column: IAA, right column: IFM): (a) and (b) - soft-tissue; (b) and (c) - adipose; (d) and (e) cortical bone; (g) and (h) - spongiosa.

However, objects-of-interest to medical imaging are not such small samples and extend over a much greater spatial volume. Diffraction peaks might be expected to 

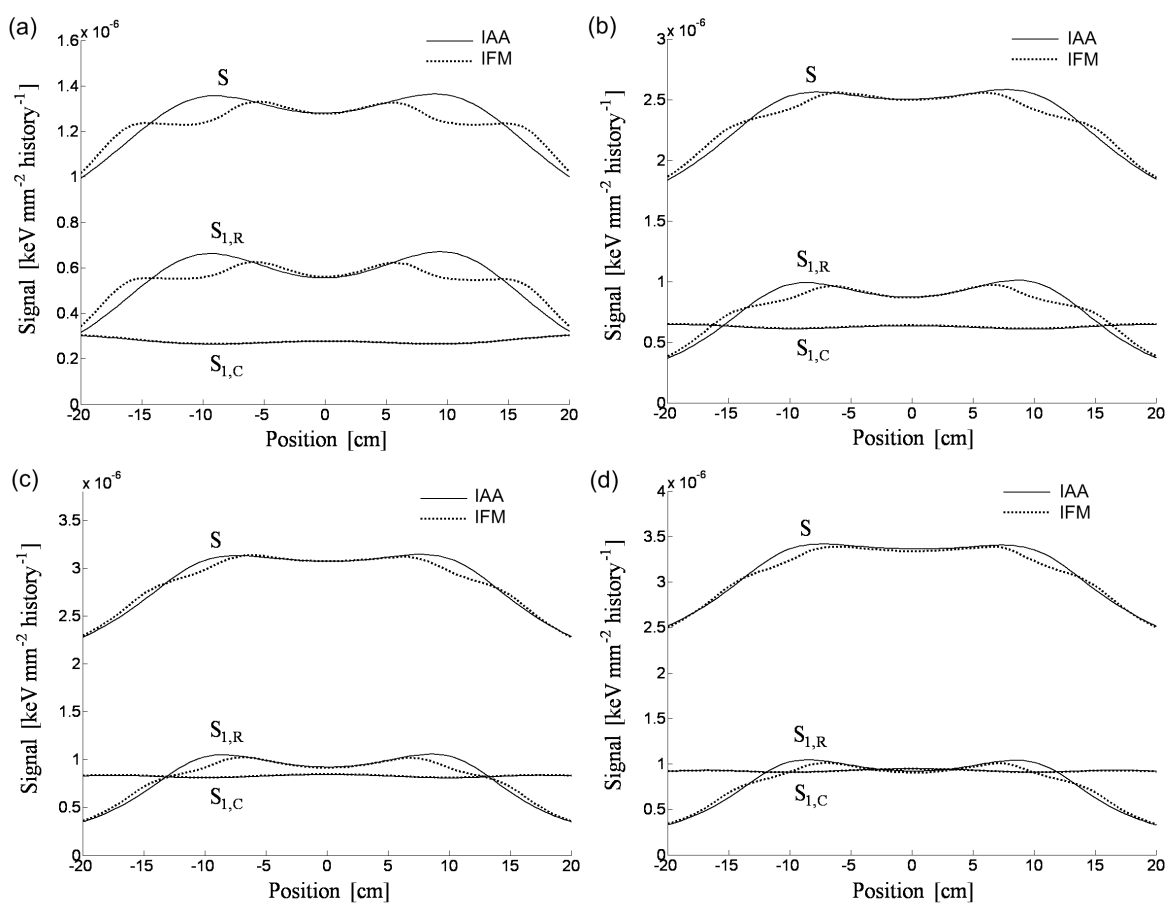

Figure 5. Line profiles of scatter signal for a $16 \mathrm{~cm}$ diameter cylinder: (a) $50 \mathrm{kV}$ x-ray beam; (b) 75 kV x-ray beam; (c) 100 kV x-ray beam; and (d) 125 kV x-ray beam.

average out in detector regions behind extended homogeneous areas. Also, the relative contribution of Compton scattering would be expected to increase for larger irradiated objects, thus diminishing the significance of any differences in the Rayleigh contribution. Figure 5 (a) shows a line-profile of scatter signal at the centre-line of the detector produced by a water cylinder of $16 \mathrm{~cm}$ diameter and length $30 \mathrm{~cm}$. This scatter was simulated for a $50 \mathrm{kV}$ x-ray beam and $10^{5}$ photon histories. The half fan-angle and half cone-angle of the beam were both set to $7.61^{\circ}$ (to exactly encompass the detector). The simulation time was approximately 3 minutes for this single line-profile of 100 points. Figures 5 (b) to (d) show line-profiles for $75 \mathrm{kV}, 100 \mathrm{kV}$ and $125 \mathrm{kV}$ beams, respectively. The IAA model produces a 'double-peak' distribution remarked on by Kyriakou et al (2008) for an identical phantom. Note, however, that the IFM predictions contain higher-spatial frequencies in the scatter signal. Differences between the IAA and IFM predictions therefore remain for this broad-area beam and extended object. The discrepancy is reduced for the higher tube potentials, but not eliminated. This suggests that the importance of inter-atomic interference may not be limited to thin samples or narrow beams. However, a cylinder of water does not accurately describe the inhomogeneity or shape of a realistic patient.

Figures 6 (a), (c) and (e) (left column) present the primary projection images for the head, thorax and pelvis phantoms, respectively. These images consist of 256x256 pixels. A $100 \mathrm{kV}$ x-ray beam was simulated for the head, while a $120 \mathrm{kV}$ beam was used for both the thorax and pelvis. This reflects clinical practice in our department. 

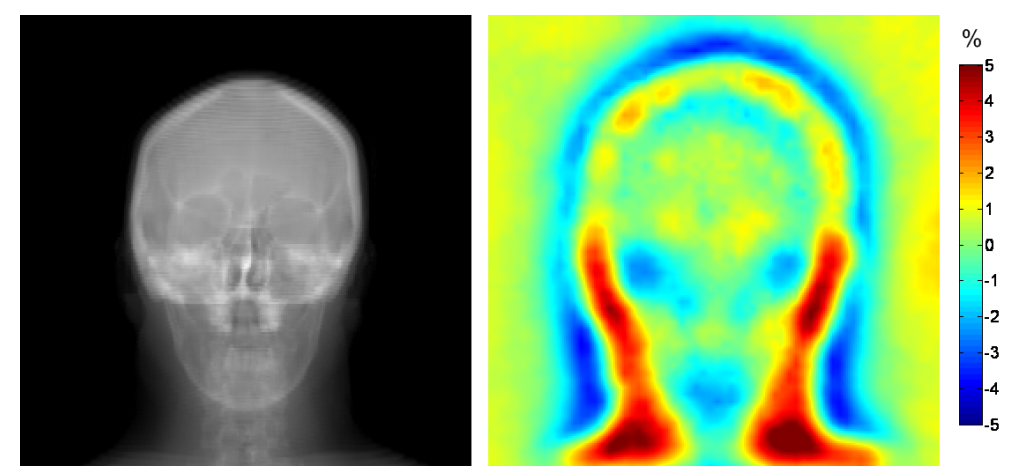

(a)

(b)
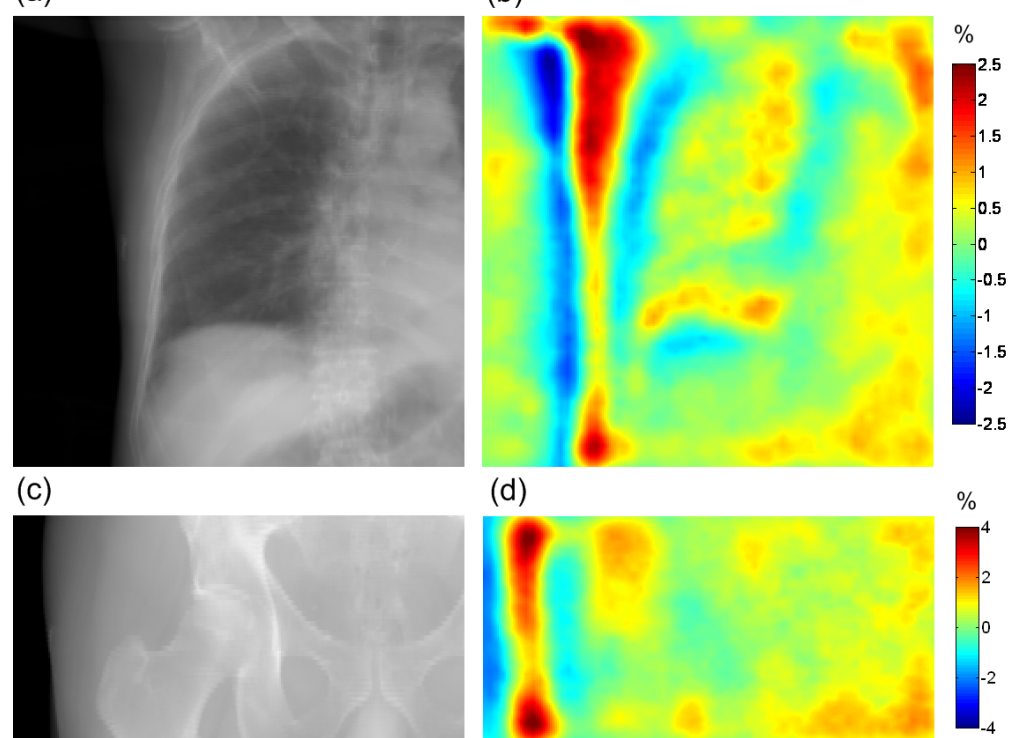

(d)

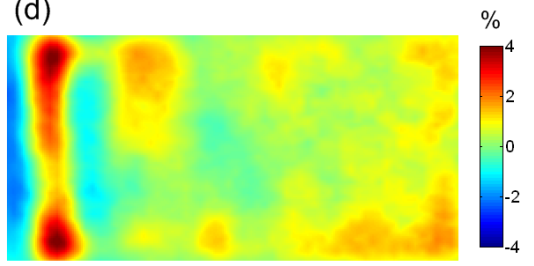

(e)

(f)

Figure 6. Projection images: (a) primary image of a skull and (b) scatter difference image for the skull (IAA - IFM); (c) primary image for a thorax and (d) scatter difference image for the thorax (IAA - IFM); (e) primary image for a pelvis and (f) scatter difference image for the pelvis (IAA - IFM). Hot regions (cold regions) indicates that IAA prediction exceeds (is less than) IFM prediction.

The differences between the scatter predictions of the IAA and IFM simulations, as a percentage of the total scatter at that point, are also shown in figures 6 (b), (d) and (f) (right column). These were calculated using $2.5 \times 10^{5}$ histories in each instance, with each simulation taking less than an hour. It should be observed that the discrepancy in some regions is as much as several percent of the total scatter at those points. Note that the largest discrepancies occur at the boundaries between materials. For example, the transition from the jaw to the neck, in figure 6 (b), or the hot regions present in the areas corresponding to the gaps between ribs in figure 6 (d). Therefore, for extended inhomogeneous objects and broad-area beams, use of the IAA can still introduce appreciable errors into scatter prediction. These are primarily close to the projected shadows of the boundaries of inhomogeneities within patients. Such errors are not large, being only a few percent of the total scatter, but would be expected to 

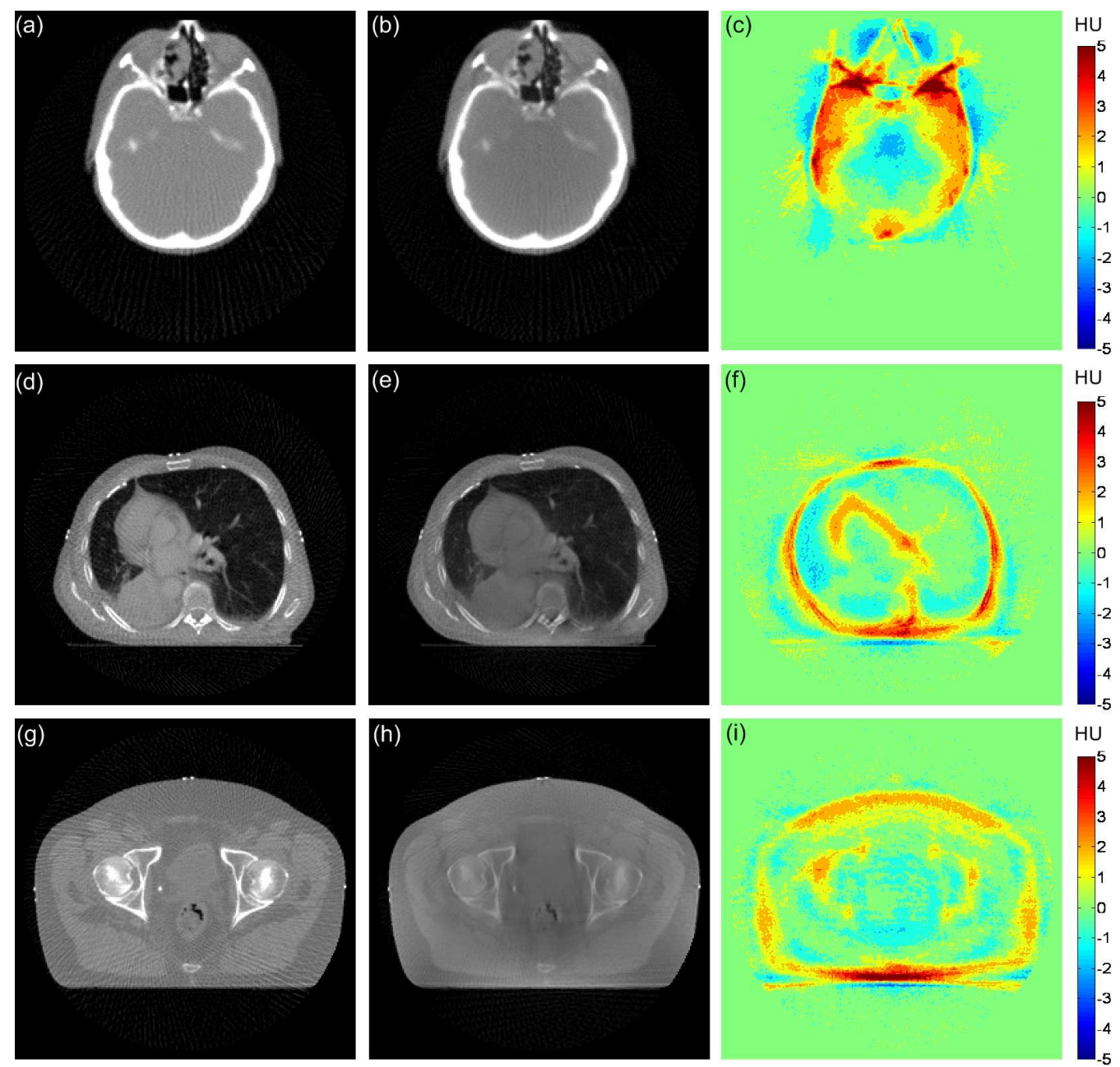

Figure 7. CT reconstructions of the head: (a)-(c); thorax: (d)-(f); and pelvis: (g)(i). The left column corresponds to reconstructions without scatter. The middle column show reconstructions with scatter (IFM approach). The right column shows the difference images between reconstructions with scatter (IAA - IFM)

increase as the tube potential is lowered.

To introduce appreciable errors into CT reconstructions, it is required that the IAA introduce sizable errors into scatter predictions. This is not sufficient, however. The degree of scatter must also be appreciable in comparison to the primary signal at the points of discrepancy. Further, it is not clear that the errors introduced into simulated projected images would have a cumulative and therefore sizable effect on reconstructed CT-number. To address this issue, a set of 180 projection images were simulated over $2 \pi$ radians for each anthropomorphic phantom. Each projection image consisted of $256 \times 256$ pixels. The scatter contribution to each pixel was calculated using $4 \times 10^{6}$ photon histories. This large number of histories was selected to eliminate any trace of stochastic noise in the images and resulted in calculation times of several hours per projection. Figures 7 (a), (d) and (g) (left column) present the images reconstructed for 
the head, thorax and pelvis, respectively, using the primary signal only. Figures 7 (b), (e) and (h) (middle column) present the same reconstructions, with the same windowing, but with scatter present in the projections (calculated in the IFM approach). The loss of contrast and global lowering of CT-number, due to the presence of scatter, is apparent. This is particularly obvious for the thorax and pelvis. Figures 6 (c), (f) and (i) (right column), show the difference images of the scatter-polluted reconstructions, calculated in the IAA and IFM approaches. Clearly the differences in reconstructed CT-number exist, due to the choice of model for Rayleigh scattering. These tend to occur close to the boundaries between materials. Notably, discrepancies occur at the boundary of the body and the surrounding air as can be seen in all three images. It can also occur internally, for example, at the boundary between the heart and lung in figure 6 (f). Despite the existence of such discrepancies, the magnitude of the differences are only of the order of a few Hounsfield Units (HU). As such, they are unlikely to be of any practical significance.

\section{Conclusion}

For the typical medical x-ray imaging detector geometry and polyenergetic source modelled in this work, the limitations of the IAA lead to large errors in the predicted spatial distribution of scatter. However, for projection images of extended anthropomorphic objects, such as the head, thorax and pelvis, the errors in the predicted scatter signal were within a few percent of the total scatter. The use of the IAA must therefore only be questioned when high quantitative accuracy is sought in the scatter contribution to individual projection images. Discrepancies, for heterogeneous objects, are generally concentrated behind regions of inhomogeneity and do not have a sizable cumulative effect in CT reconstruction. The IAA can then be considered sufficient for modelling scatter-correction in CT imaging.

\section{Acknowledgments}

This work was partially supported by research grant C46/A2131 from Cancer Research UK. We acknowledge NIHR funding to the NHS Biomedical Research Centre.

\section{References}

Allison J, Amako K, Apostolakis J, Araujo H, Dubois PA, Asai M, Barrand G, Capra R, Chauvie S, Chytracek R, Cirrone GAP, Cooperman G, Cosmo G, Cuttone G, Daquino GG, Donszelmann M, Dressel M, Folger G, Foppiano F, Generowicz J, Grichine V, Guatelli S, Gumplinger P, Heikkinen A, Hrivnacova I, Howard A, Incerti S, Ivanchenko V, Johnson T, Jones F, Koi T, Kokoulin R, Kossov M, Kurashige H, Lara V, Larsson S, Lei F, Link O, Longo F, Maire M, Mantero A, Mascialino B, McLaren I, Lorenzo PM, Minamimoto K, Murakami K, Nieminen P, Pandola L, Parlati S, Peralta L, Perl J, Pfeiffer A, Pia MG, Ribon A, Rodrigues P, Russo G, Sadilov S, Santin G, Sasaki T, Smith D, Starkov N, Tanaka S, Tcherniaev E, Tome B, Trindade A, Truscott P, Urban L, 
Verderi M, Walkden A, Wellisch JP, Williams DC, Wright D, Yoshida H Geant4 developments and applications IEEE Trans. Nucl. Sci. 53(1) 270-278.

Berger MJ, Hubbell JH, Seltzer SM, Chang J, Coursey JS, Sukumar R and Zucker DS 2005 XCOM: Photon Cross Section Database version 1.3 (Gaithersburg, MD, US: NIST).

Cardoso SC, Goncalves OD, Schechter H and Eichler J 2003 Modelling the elastic scattering in diagnostic radiology: the importance of structure form factors Phys. Med. Biol. 48(13) 1907-1916.

Chan H-P and Doi K 1983 The validity of Monte Carlo simulation in studies of scattered radiation in diagnostic radiology Phys. Med. Biol. 28(2) 109-29.

Elshemey WM, Elsayed AA and El-Lakkani A 1999 Characteristic of low-angle scattering from some biological samples Phys. Med. Biol. 44(12) 2907-2915.

Feldkamp L A, Davis L C and Kress J W 1984 Practical cone-beam algorithm J. Opt. Soc. Am. A 1 612-19.

Guinier A 1994 X-ray diffraction in crystals, imperfect crystals, and amorphous bodies (New York: Dover Publications, Inc.) Chapter 3.

Harding G and Schreiber B 1999 Coherent X-ray scatter imaging and its applications in biomedical science and industry Rad. Phys. Chem. 56 229-245.

Head-Gordon T and Hura G 2002 Water structure from scattering experiments and simulation Chem. Rev. 102 2651-2670.

Hubbell J H, Veigele J W, Briggs E A, Brown R T, Cromer D T, Howerton R J 1975 Atomic Form factors, Incoherent Scattering Functions, and Photon Scattering Cross Sections JPCRD 4(3) 471538.

International Commission on Radiation Units and Measurements 1988 ICRP Report 44: tissue substitutes in radiation dosimetry and measurement (Bethesda, MD: ICRU).

Johns PC and Yaffe MJ 1983 Coherent scatter in diagnostic radiology Med. Phys. 10(1) 40-50.

Kawrakow I and Rogers D W O 2003 NRCC Report PIRS-701: the EGSnrc Code System (Ottowa, Canada: NRCC).

Kosanetzky J, Knoerr B, Harding G, Neitzel U 1987 X-ray diffraction measurements of some plastic materials and body tissues Med. Phys. 14(4) 526-532.

Kyriakou Y, Meyer M and Kalender W A 2008 Technical note: comparing coherent and incoherent scatter effects for cone-beam CT Phys. Med. Biol. 53(10) N175-N185.

Morin LRM 1982 Molecular form factors and photon coherent scattering cross sections of water J. Phys. Chem. Ref. Data 11(4) 1091-1098.

Narten AH and Levy HA 1971 Liquid water - molecular correlation functions from x-ray diffraction 55(5) 2263-2269.

Peplow DE and Verghese K 1998 Measured molecular coherent scattering form factors of animal tissues, plastics and human breast tissue Phys. Med. Biol. 43(9) 2431-2452.

Peplow DE and Verghese K 2000 Digital mammography image simulation using Monte Carlo 27(3) 568-579.

Persliden J and Alm Carlsson G 1997 Scatter rejection by air gaps in diagnostic radiology. Calculations using a Monte carlo collision density method and consideration of molecular interference in coherent scattering Phys. Med. Biol. 42(1) 155-175.

Poludniowski GG 2007 Calculation of $x$-ray spectra emerging from an x-ray tube. Part II. X-ray production and filtration in x-ray targets Med. Phys. 34(6) 2175-76.

Poludniowski GG, Evans PM and Webb S 2009a An efficient Monte Carlo-based algorithm for scatter correction in keV cone-beam CT Phys. Med. Biol. 54(12) 3847-3864.

Poludniowski GG, Landry G, Deblois F, Evans PM and Verhaegen F 2009b SpekCalc: a program to calculate photon spectra from tungsten anode x-ray tubes Phys. Med. Biol. 54(19) N433-8.

Roberts DA, Hansen V N, Niven A C, Thompson M G, Seco J and Evans P M 2008 A low Z linac and flat panel imager: comparison with the conventional imaging approach Phys. Med. Biol. 53(22) 6305-19.

Rogers D W O, Ma C-M, Ding G X, Walters B, Sheikh-Bagheri D, and Zhang GG 2004 NRCC Report 
Royle GJ and Speller RD 1991 Low angle x-ray scattering for bone analysis Phys. Med. Biol. 36(3) 383-389.

Salvat F, Fernandez-Varea J M, Acosta E and Sempau J 2003 PENELOPE, A Code System for Monte Carlo Simulation of Electron and Photon Transport (OECD Nuclear Energy Agency, Issy-lesMoulineaux, France)

Siewerdsen J H and Jaffray D A 2001 Cone-beam computed tomography with a flat-panel imager: Magnitude and effects of x-ray scatter Med. Phys. 28(2) 220-31.

Soper AK 2000 The radial distribution functions of water and ice from 220 to 673 $\mathrm{K}$ and at pressures up to $400 \mathrm{MPa}$ Chem. Phys. 258 121-137.

Taibi A, Royle GJ and Speller RD 2000 A Monte Carlo simulation study to investigate the potential of diffraction enhanced breast imaging IEEE Trans. Nucl. Sci. 47 1581-1586.

Tartari A, Taibi A, Bonifazzi C and Baraldi C 2002 Updating of form factor tabulations for coherent scattering of photons in tissues Phys. med. biol. 47(1) 163-175.

Waters LS, McKinney GW, Durkee JW, Fensin ML, Hendricks JS, James MR, Johns RC, Pelowitz DB 2007 The MCNPX Monte Carlo Radiation Transport Code HADRONIC SHOWER SIMULATION WORKSHOP. AIP Conference Proceedings 896 81-90. 